\title{
Integrability of the Rabi Model
}

\author{
D. Braak \\ EP VI and Center for Electronic Correlations and Magnetism, University of Augsburg, 86135 Augsburg, Germany
} (Received 22 April 2011; published 29 August 2011)

\begin{abstract}
The Rabi model is a paradigm for interacting quantum systems. It couples a bosonic mode to the smallest possible quantum model, a two-level system. I present the analytical solution which allows us to consider the question of integrability for quantum systems that do not possess a classical limit. A criterion for quantum integrability is proposed which shows that the Rabi model is integrable due to the presence of a discrete symmetry. Moreover, I introduce a generalization with no symmetries; the generalized Rabi model is the first example of a nonintegrable but exactly solvable system.
\end{abstract}

DOI: 10.1103/PhysRevLett.107.100401

PACS numbers: 03.65.Ge, 02.30.Ik, 42.50.Pq

The Rabi or single-mode spin-boson model constitutes probably the simplest physical system beyond the harmonic oscillator. Introduced over 70 years ago [1], its applications range from quantum optics [2] and magnetic resonance to solid state [3] and molecular physics [4]. Very recently, it has gained a prominent role in novel fields of research such as cavity QED [5] and circuit QED [6]. It can be experimentally realized in Josephson junctions [7] or using trapped ions [8], in Cooper-pair boxes [9] and flux $q$-bits [10]. In this way, its complete theoretical understanding is mandatory for all feasible approaches to quantum computing [11]. Despite its old age and central importance, the Rabi model has not been exactly solved [3,12-15]. With the other paradigm of quantum physics, the hydrogen atom, it shares an infinite-dimensional state space but-in contrast to the latter-the spectrum and eigenfunctions of the Rabi model are known only by numerical diagonalization in a truncated, finitedimensional Hilbert space. This is quite surprising, as the Rabi model has a smaller number of degrees of freedom (d.o.f.) than the hydrogen atom. In particular, a single d.o.f., subject to a harmonic potential, couples to a quantum system with only two allowed states $|\uparrow\rangle$ and $|\downarrow\rangle$. Therefore, it does not possess a classical limit: the quantum d.o.f. has a finite-dimensional Hilbert space and places the Rabi model in between the case of one and two (classical) d.o.f. The Hamiltonian reads $(\hbar=1)$

$$
H_{R}=\omega a^{\dagger} a+g \sigma_{x}\left(a+a^{\dagger}\right)+\Delta \sigma_{z} .
$$

Here, the $\sigma_{x, z}$ are Pauli matrices for the two-level system with level splitting $2 \Delta$ and $a\left(a^{\dagger}\right)$ denote destruction (creation) operators of a single bosonic mode with frequency $\omega$. These two systems are coupled through a term proportional to $g$, which has different interpretations according to the experimental situation to model.

Although (1) represents the simplest of all physically sensible interacting quantum systems, it poses a serious obstruction to its analytical solution because of the apparent lack of a second conserved quantity besides the energy, which has led to the widespread opinion that it cannot be integrable [16-21]. To remedy this difficulty, Jaynes and Cummings (JC) proposed in the 1960s an approximation to (1) which does possess such a quantity [22]. Their Hamiltonian reads

$$
H_{\mathrm{JC}}=\omega a^{\dagger} a+g\left(\sigma^{+} a+\sigma^{-} a^{\dagger}\right)+\Delta \sigma_{z},
$$

with $\sigma^{ \pm}=\left(\sigma_{x} \pm i \sigma_{y}\right) / 2$. Here, the operator $C=a^{\dagger} a+$ $\frac{1}{2}\left(\sigma_{z}+1\right)$ commutes with $H_{\mathrm{JC}}$ and leads at once to the solvability of (2). The JC model is the so-called "rotatingwave" approximation to (1) and was justified because the conditions of near resonance $2 \Delta \approx \omega$ and weak coupling $g \ll \omega$ for such an approximation are realized in many experiments. The conservation of $C$ signifies that the state space decomposes into an infinite sum of two-dimensional invariant subspaces. Each eigenstate of (2) is then labeled by $C=0,1,2, \ldots$ and a two-valued index, for example + and - , denoting a basis vector in the two-dimensional subspace which belongs to $C$. Whereas the possible values of $C$ form an unbounded set, corresponding to the quantization of a classical d.o.f., the second quantum number can take only two values, reflecting the intrinsic quantum nature of the two-level system.

The conserved quantity $C$ generates a continuous $U(1)$ symmetry of the JC model (2) which is broken down to $\mathbb{Z}_{2}$ in the Rabi model (1) due to the presence of the term $a^{\dagger} \sigma^{+}+$ $a \sigma^{-}$[14]. This residual $\mathbb{Z}_{2}$ symmetry, usually called parity, leads to a decomposition of the state space into just two subspaces $\mathcal{H}_{ \pm}$, each with infinite dimension. One would conclude that this symmetry cannot suffice to solve the model exactly-but in fact it does. Whereas a discrete symmetry is too weak to accommodate a classical (continuous) d.o.f., it can do so with a quantum d.o.f. We observe a direct relation between the nature of the d.o.f. (continuous or discrete) and the symmetry $\left[U(1)\right.$ versus $\left.\mathbb{Z}_{2}\right]$, which can be used to "eliminate" it by fixing the corresponding irreducible representation.

Our main result is the following. The spectrum of (1) consists of two parts, the regular and the exceptional spectrum. Almost all eigenvalues are regular and given by the zeros of the transcendental function $G_{ \pm}(x)$ in the 
variable $x$, which is defined through its power series in the coupling $g$ :

$$
G_{ \pm}(x)=\sum_{n=0}^{\infty} K_{n}(x)\left[1 \mp \frac{\Delta}{x-n \omega}\right]\left(\frac{g}{\omega}\right)^{n} .
$$

The coefficients $K_{n}(x)$ are defined recursively,

$$
n K_{n}=f_{n-1}(x) K_{n-1}-K_{n-2},
$$

with the initial condition $K_{0}=1, K_{1}(x)=f_{0}(x)$, and

$$
f_{n}(x)=\frac{2 g}{\omega}+\frac{1}{2 g}\left(n \omega-x+\frac{\Delta^{2}}{x-n \omega}\right) .
$$

To derive the result (3)-(5) for $G_{ \pm}(x)$ [23], we used the representation of bosonic operators in the Bargmann space of analytical functions [24].

The function $G_{ \pm}(x)$ is not analytic in $x$ but has simple poles for $x=0, \omega, 2 \omega, \ldots$ (see Fig. 1); these poles are precisely the eigenvalues of the uncoupled bosonic mode. Then the regular energy spectrum of the Rabi model in each invariant subspace $\mathcal{H}_{ \pm}$with parity \pm 1 is given by the zeros of $G_{ \pm}(x)$ : for all values $x_{n}^{ \pm}$with $G_{ \pm}\left(x_{n}^{ \pm}\right)=0$, the $n$th eigenenergy with parity \pm 1 reads $E_{n}^{ \pm}=x_{n}^{ \pm}-g^{2} / \omega$.

For special values of model parameters $g, \Delta$, there are eigenvalues which do not correspond to zeros of (3); these are the exceptional ones. All exceptional eigenvalues have the form $E_{n}^{e}=n \omega-g^{2} / \omega$, and the necessary and sufficient condition for the occurrence of the eigenvalue $E_{n}^{e}$ reads $K_{n}(n \omega)=0$, which furnishes a condition on the model parameters $g$ and $|\Delta|$. These exceptional solutions to (1) have been known for a long time and were first discovered by Judd [25]. They occur when the pole of $G_{ \pm}(x)$ at $x_{n}=n \omega$ is lifted because its numerator in (3) vanishes. As then $G_{ \pm}(x) \neq 0$, this eigenvalue has no definite parity and is therefore doubly degenerate.

The functional form of $G_{ \pm}(x)$ reads

$$
G_{ \pm}(x)=G_{ \pm}^{0}(x)+\sum_{n=0}^{\infty} \frac{h_{n}^{ \pm}}{x-n \omega},
$$

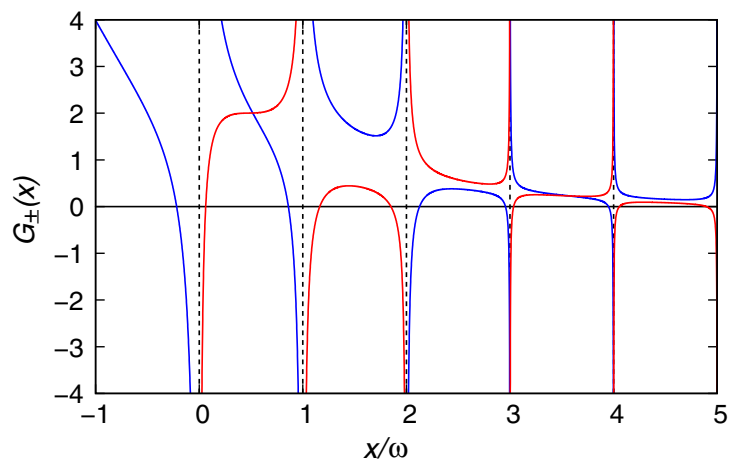

FIG. 1 (color online). $G_{+}(x)$ [light gray (red) lines] and $G_{-}(x)$ [dark gray (blue) lines] in the interval $[-1,5]$ for $\omega=1$, $g=0.7$, and $\Delta=0.4$ where $G_{ \pm}^{0}(x)$ is entire in $x$. The position of the solutions to $G_{ \pm}(x)=0$ is dictated by the pole structure of $G_{ \pm}(x)$, which leads to the conjecture that the number of eigenvalues in each interval $[n \omega,(n+1) \omega]$ is restricted to be 0,1 , or 2. Moreover, an interval $[n \omega,(n+1) \omega]$ with two roots of $G_{ \pm}(x)=0$ can only be adjacent to an interval with one or zero roots; in the same way, an empty interval can never be adjacent to another empty interval. These conjectures, which can be confirmed numerically, lead to a fairly regular distribution of the energies and a violation of the BerryTabor criterion $[18,26]$. Figure 2 shows the lowest part of the Rabi spectrum as function of $g$. There are no level crossings within each parity subspace, allowing the unique labeling of each state $|\psi\rangle$ with a pair of two quantum numbers, $|\psi\rangle=\left|n_{0}, n_{1}\right\rangle$ : the parity quantum number $n_{0}$, which takes the values +1 and -1 , and $n_{1}=0,1,2, \ldots$, which denotes the $n_{1}$ th zero of $G_{n_{0}}(x)$. The exceptional solutions correspond to level crossings between $\mathcal{H}_{+}$and $\mathcal{H}_{-}$. This characterization of each eigenstate through two quantum numbers corresponding to the d.o.f. of the system parallels the unique assignment of three quantum numbers $n, l, m$ to the eigenstates of the hydrogen atom, reflecting the quantization of radial and angular d.o.f., a hallmark of integrability.

It seems therefore natural to call a quantum system integrable when such an assignment can be made-independent of the explicit determination of conserved quantities or even action variables, which is only possible if the system under consideration has an integrable classical limit in the sense of Liouville. I propose the following criterion.

Criterion of quantum integrability. - If each eigenstate of a quantum system with $f_{1}$ discrete and $f_{2}$ continuous

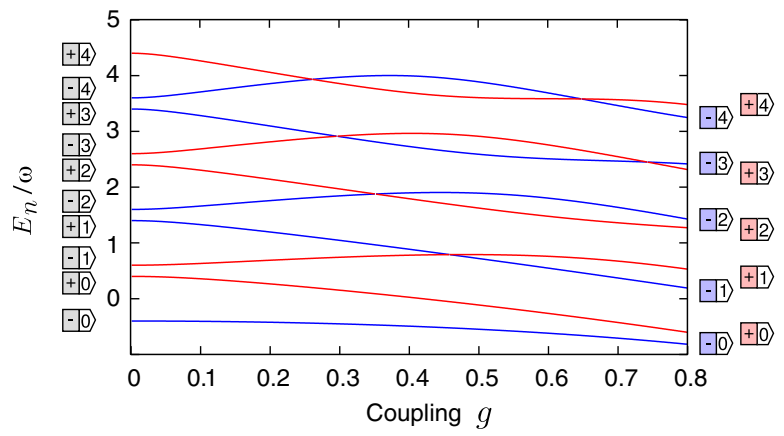

FIG. 2 (color online). Rabi spectrum for $\Delta=0.4, \omega=1$, and $0 \leq g \leq 0.8$ in the spaces with positive [light gray (red) lines] and negative [dark gray (blue) lines] parity. Within each space the states are labeled with ascending numbers $0,1,2, \ldots$ This labeling does not change with $g$ because no lines intersect within spaces of fixed parity. The spectral graph is composed of two intersecting "ladders" of level lines, each corresponding to one parity subspace. This labeling is used on the right side of the figure. On the left side the states with $g=0$ are labeled by the uncoupled d.o.f.; i.e., in $| \pm, n\rangle$, + or - corresponds to the twolevel system and $n=0,1,2, \ldots$ to the eigenstates of the bosonic mode. 
d.o.f. can be uniquely labeled by $f_{1}+f_{2}=f$ quantum numbers $\left\{d_{1}, \ldots, d_{f_{1}}, c_{1}, \ldots c_{f_{2}}\right\}$, such that the $d_{j}$ can take on $\operatorname{dim}\left(\mathcal{H}_{j}\right)$ different values, where $\mathcal{H}_{j}$ is the state space of the $j$ th discrete d.o.f. and the $c_{k}$ range from 0 to infinity, then this system is quantum integrable.

The criterion does not presuppose the existence of a family of commuting operators whose different spectra are associated with the $\left\{d_{j}, c_{k}\right\}$ but provides a phenomenological condition on the spectral graph of the system, that is, the spectrum as function of a parameter, typically one of the coupling constants. Without such a deformation parameter (which must conserve integrability), the association of more than one quantum number to the levels of a nondegenerate spectrum is ill-defined and would be restricted either to models solvable via Bethe ansatz [27] or systems with integrable classical limit. As is well known, the Berry-Tabor criterion [26] relies precisely on the existence of this limit and fails for models of the Rabi or Jaynes-Cummings type.

According to the proposed criterion, integrability is equivalent to the existence of $f$ numbers to classify eigenstates uniquely. It should be emphasized that these "quantum numbers" are a more general concept than the radial and angular quantum numbers known from atomic physics because they are only defined with respect to the unique description of eigenstates: the integrable systems differ from the nonintegrable because they allow for a "finegrained" description through an $f$-dimensional vectorial label, whereas the latter have a one-dimensional label corresponding to energy as the only conserved quantity. While a direct application to models with more than two continuous d.o.f. is difficult, it allows us to define integrability for the class of models in the quantum limit with less than two classical d.o.f. which comprises many systems of physical importance, e.g., the Dicke and Tavis-Cummings models [28].

The Rabi model has $f_{1}=f_{2}=1$ and degeneracies take place between levels of states with different parity, whereas within the parity subspaces no level crossings occur. The spectral graph consists of two "ladders" of level lines $\left|n_{0}, m\right\rangle$. Each ladder corresponds to an invariant subspace of the $\mathbb{Z}_{2}$ symmetry characterized by $n_{0}= \pm$, the parity eigenvalue. The global label (valid for all values of $g$ ) is two dimensional as $f=f_{1}+f_{2}=2$; the Rabi model belongs therefore to the class of integrable systems.

It may be, however, that the symmetry is even stronger than necessary to achieve integrability, analogous to the classically "superintegrable" systems [29]. The JC model is an example for this case. Here, the continuous $U(1)$ symmetry leads to a further decomposition of the subspaces with fixed parity. The larger number of dynamically decoupled state spaces entails a second possibility to label the states uniquely: through $C$ and a two-valued index $n_{0}= \pm$. Now, all levels with different $C$ may intersect, leading not to just two but infinitely many ladders labeled

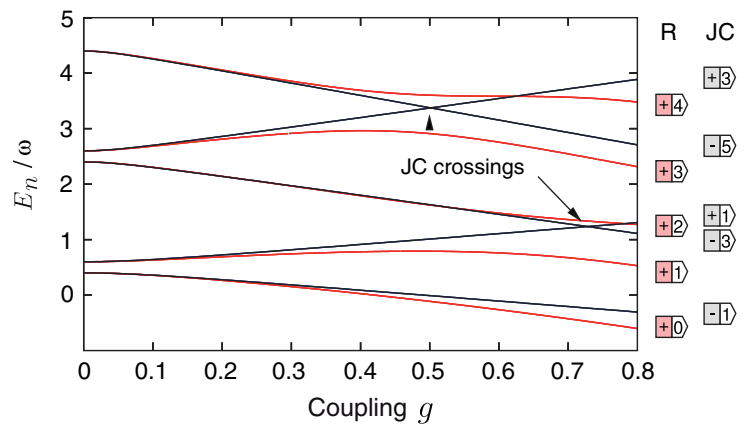

FIG. 3 (color online). The spectrum of the Jaynes-Cummings model (black lines) compared with the Rabi model [gray (red) lines] for $\Delta=0.4$ and even parity. The state labeling of the former has the form $|\psi\rangle=\left|n_{0}, C\right\rangle$ with $n_{0}= \pm$. Two accidental crossings occur between levels with $C=5$ and $C=3$ at $g \approx 0.5$ and between $C=1$ and $C=3$ at $g \approx 0.73$.

by $C$, each with two rungs, labeled by $n_{0}$. In Fig. 3 the four lowest levels of the Rabi model with positive parity are compared with the corresponding levels of the JC model with $C=1,3,5$. The enlarged symmetry of the latter leads to two level crossings which are not present in the Rabi model [30]. The appearance of intersecting ladders in the spectral graph can be detected without knowledge of the exact solution or the correct assignment of quantum numbers to the different levels, the only condition being a sufficient numerical resolution to discern degeneracies from narrow avoided crossings. This is a phenomenological virtue of the proposed criterion which could be used in computer experiments to test whether a given numerically solvable system possesses a hidden integrable structure.

On the other hand, the absence of any level crossings in the spectral graph is sufficient for nonintegrability if the total number of d.o.f. exceeds one: It means that the states can be classified only by energy, the single conserved quantity always present in Hamiltonian systems, and no invariant subspaces exists. In the context of the Rabi model this case can be realized by breaking the $\mathbb{Z}_{2}$ symmetry. A possible generalization of (1) reads

$$
H_{\epsilon}=\omega a^{\dagger} a+g \sigma_{x}\left(a+a^{\dagger}\right)+\epsilon \sigma_{x}+\Delta \sigma_{z}
$$

The term $\epsilon \sigma_{x}$ breaks the parity symmetry which couples the bosonic mode and the two-level system. Physically it corresponds to a spontaneous transition of the two-level system which is not driven by the radiation field. The state space does not separate into two subspaces, and indeed the spectral graph exhibits no level crossings at all if $\epsilon$ is not a multiple of $\omega / 2$ [31]. This is shown in Fig. 4. In this situation, the eigenstates can be uniquely numbered as belonging to the $n$th energy level in ascending order, $|\psi\rangle=|n\rangle$. We have only one quantum number, energy, corresponding to the sole conserved quantity. Because the number of d.o.f. nevertheless exceeds one, this model must be considered nonintegrable. Interestingly, (7) is still 


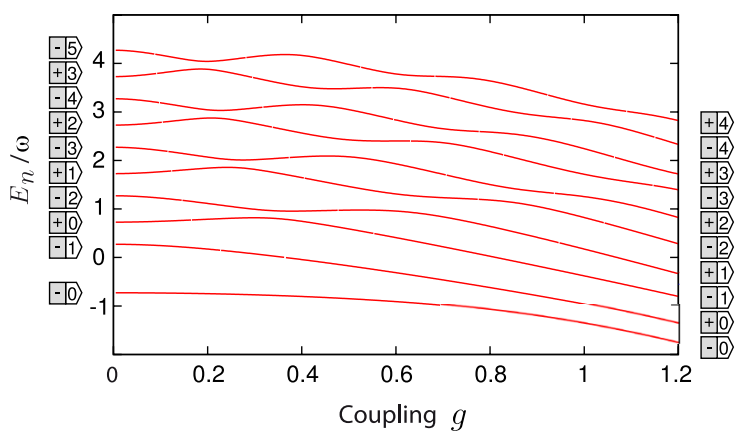

FIG. 4 (color online). The spectrum of the generalized Rabi model with broken $\mathbb{Z}_{2}$ symmetry and $\Delta=0.7, \epsilon=0.2$. The twofold labeling of the states corresponds to the two integrable limits $g=0$ on the left and $g \rightarrow \infty$ on the right. For finite $g$ neither labeling classifies the states properly.

exactly solvable, although it does not possess any symmetry [23]. Define the functions

$$
\begin{gathered}
R^{ \pm}(x)=\sum_{n=0}^{\infty} K_{n}^{ \pm}(x)\left(\frac{g}{\omega}\right)^{n}, \\
\bar{R}^{ \pm}(x)=\sum_{n=0}^{\infty} \frac{K_{n}^{ \pm}(x)}{x-n \omega \pm \epsilon}\left(\frac{g}{\omega}\right)^{n} .
\end{gathered}
$$

The $K_{n}^{ \pm}(x)$ are again recursively defined,

$$
n K_{n}^{ \pm}=f_{n-1}^{ \pm}(x) K_{n-1}^{ \pm}-K_{n-2}^{ \pm},
$$

with the initial condition $K_{0}^{ \pm}=1, K_{1}^{ \pm}(x)=f_{0}^{ \pm}(x)$, and

$$
f_{n}^{ \pm}(x)=\frac{2 g}{\omega}+\frac{1}{2 g}\left(n \omega-x \pm \epsilon+\frac{\Delta^{2}}{x-n \omega \pm \epsilon}\right) .
$$

The $n$th eigenvalue $E_{n}$ of (7) is given by the $n$th zero $x_{n}$ of

$$
G_{\epsilon}(x)=\Delta^{2} \bar{R}^{+}(x) \bar{R}^{-}(x)-R^{+}(x) R^{-}(x)
$$

through $E_{n}=x_{n}-g^{2} / \omega$. The fact that $H_{\epsilon}$ can be diagonalized analytically although not even a discrete symmetry is present signifies that integrability and solvability are not equivalent in the realm of quantum physics. In contrast to classical mechanics, nonintegrable quantum systems with exact solutions exist.

I wish to thank K.-H. Höck, N. Andrei, M. Dzierzawa, S. Graser, and especially T. Kopp for stimulating discussions. This work was supported by the DFG through TRR 80 .

[1] I. I. Rabi, Phys. Rev. 49, 324 (1936); 51, 652 (1937).

[2] V. Vedral, Modern Foundations of Quantum Optics (Imperial College Press, London, 2006).
[3] E. K. Irish, Phys. Rev. Lett. 99, 173601 (2007).

[4] I. Thanopulos, E. Paspalakis, and Z. Kis, Chem. Phys. Lett. 390, 228 (2004).

[5] D. Englund et al., Nature (London) 450, 857 (2007).

[6] T. Niemczyk et al., Nature Phys. 6, 772 (2010).

[7] A. T. Sornborger, A. N. Cleland, and M. R. Geller, Phys. Rev. A 70, 052315 (2004).

[8] D. Leibfried, R. Blatt, C. Monroe, and D. Wineland, Rev. Mod. Phys. 75, 281 (2003).

[9] A. Wallraff et al., Nature (London) 431, 162 (2004).

[10] P. Forn-Diaz et al., Phys. Rev. Lett. 105, 237001 (2010).

[11] T. Pellizzari, S. A. Gardiner, J. I. Cirac, and P. Zoller, Phys. Rev. Lett. 75, 3788 (1995).

[12] J. Larson, Phys. Scr. 76, 146 (2007).

[13] T. Werlang, A.V. Dodonov, E.I. Duzzioni, and C.J. Villas-Bôas, Phys. Rev. A 78, 053805 (2008).

[14] L. Amico, H. Frahm, A. Osterloh, and G. A.P. Ribeiro, Nucl. Phys. B787, 283 (2007).

[15] L. Amico, H. Frahm, A. Osterloh, and T. Wirth, Nucl. Phys. B839, 604 (2010).

[16] P. W. Milonni, J. R. Ackerhalt, and H. W. Galbraith, Phys. Rev. Lett. 50, 966 (1983).

[17] R. Graham and M. Höhnerbach, Phys. Lett. A 101, 61 (1984).

[18] M. Kuś, Phys. Rev. Lett. 54, 1343 (1985).

[19] L. Bonci, R. Roncaglia, B. J. West, and P. Grigolini, Phys. Rev. Lett. 67, 2593 (1991).

[20] T. Fukuo, T. Ogawa, and K. Nakamura, Phys. Rev. A 58, 3293 (1998).

[21] C. Emary and T. Brandes, Phys. Rev. Lett. 90, 044101 (2003).

[22] E. T. Jaynes and F.W. Cummings, Proc. IEEE 51, 89 (1963).

[23] See Supplemental Material at http://link.aps.org/ supplemental/10.1103/PhysRevLett.107.100401 for a detailed derivation of Eqs. (3)-(5) and (8)-(12).

[24] V. Bargmann, Commun. Pure Appl. Math. 14, 187 (1961).

[25] B. R. Judd, J. Phys. C 12, 1685 (1979); M. Kuś, J. Math. Phys. (N.Y.) 26, 2792 (1985); M. Szopa, G. Mýs, and A. Ceulemans, J. Math. Phys. (N.Y.) 37, 5402 (1996); R. Koç, M. Koca, and H. Tütünküler, J. Phys. A 35, 9425 (2002); C. Emary and E. F. Bishop, J. Math. Phys. (N.Y.) 43, 3916 (2002).

[26] M. V. Berry and M. Tabor, Proc. R. Soc. A 356, 375 (1977).

[27] N. Andrei, arXiv:cond-mat/9408101.

[28] R. H. Dicke, Phys. Rev. 93, 99 (1954); M. Tavis and F. W. Cummings, Phys. Rev. 170, 379 (1968).

[29] N. W. Evans, Phys. Rev. A 41, 5666 (1990).

[30] V. V. Stepanov, G. Müller, and J. Stolze, Phys. Rev. E 77, 0066202 (2008).

[31] If $\epsilon$ is an integer multiple of $\omega / 2$, the model becomes integrable again and concomitant level crossings appear. 